\title{
Efficacy and factors affecting the choice of enucleation and liver resection for giant hemangioma: a retrospective propensity score-matched study
}

\author{
Mingguang Ju, Feng Xu, Wenyan Zhao and Chaoliu Dai ${ }^{*}$
}

\begin{abstract}
Background: Liver resection (LR) and enucleation (EN) are the main surgical treatment for giant hepatic hemangioma $(\mathrm{HH})$, but how to choose the type of surgery is still controversial. This study aimed to explore the efficacy and the factors affecting the choice of open procedure for $\mathrm{HH}$.

Methods: The data for patients with pathologically confirmed HH who underwent open surgery from April 2014 to August 2020 were analyzed retrospectively. Univariate and multivariate analyses with logistic regression were performed to disclose the factors associated with the choice of EN or LR. Propensity score matching (PSM) analysis was used to compare the efficacy of the two procedures.

Results: A total of 163 and 110 patients were enrolled in the EN and LR groups. Following 1:1 matching by PSM analysis, 66 patients were selected from each group. Centrally located lesions (OR: $0.131,95 \% \mathrm{Cl} 0.070-0.244$ ), tumors size $>12.1 \mathrm{~cm}$ (OR: $0.226,95 \% \mathrm{Cl} 0.116-0.439)$ and multiple tumors (OR: 1.860, 95\% Cl 1.003-3.449) were independent factors affecting the choice of EN. There was no significant difference in the median operation time (156 vs. $195 \mathrm{~min}$, $P=0.156)$, median blood loss ( 200 vs. $220 \mathrm{ml}, \mathrm{P}=0.423)$, blood transfusion rate ( $33.3 \%$ vs. $33.3 \%, P=1.000)$, mean postoperative feeding ( 3.1 vs. $3.3 \mathrm{~d}, \mathrm{P}=0.460)$, mean postoperative hospital stay ( $9.5 \mathrm{vs}$. $9.0 \mathrm{~d}, \mathrm{P}=0.206)$, or the major complication rates between the two groups.
\end{abstract}

Conclusions: Peripherally located lesions, tumors size $\leq 12.1 \mathrm{~cm}$ and multiple tumors were more inclined to receive EN. There was no significant difference in the efficacy of EN or LR.

Keywords: Hepatic hemangioma, Enucleation, Liver resection, Propensity score matching, Surgery

\section{Background}

Hepatic hemangioma $(\mathrm{HH})$ is the most common benign tumor of the liver [1]. Most patients with $\mathrm{HH}$ are asymptomatic and the lesions are incidentally detected on ultrasound, computed tomography (CT) or magnetic resonance imaging (MRI) [2]. Small asymptomatic $\mathrm{HH}$

\footnotetext{
*Correspondence: cmudaichaoliu@163.com

Department of General Surgery, Shengjing Hospital of China Medical University, No.36 Sanhao Street, Heping District, Shenyang 110004, Liaoning, China
}

(generally $<5 \mathrm{~cm}$ ) can be followed up without treatment. However, some patients with giant $\mathrm{HH}(\geq 5 \mathrm{~cm})$ may develop clinical symptoms or complications such as abdominal pain, jaundice, nausea, vomiting. A small number of patients may develop Kasabach-Merritt syndrome due to platelet destruction in the hemangioma or spontaneous rupture [3-6].

Surgical resection is the main treatment strategy for symptomatic giant $\mathrm{HH}$ [7-9]. The surgical treatment for HH includes open, laparoscopic, or robotic liver resection (LR) and enucleation (EN) [2, 10]. EN was first 
proposed in 1988 [11]. Some surgeons think that there are few blood vessels in the interface between $\mathrm{HH}$ and liver parenchyma, and it can be bluntly separated along with the interface so the purpose of less bleeding and complete removal of the focus can be achieved, which means EN is simpler and safer than the traditional LR [12-14], especially for $\mathrm{HH}$ at special locations, such as tumors near the hepatic hilum [15]. However, some studies found that there was no significant difference in the curative effect or postoperative outcome between the two techniques [16-18]. The characteristics of tumors in the previous studies were quite different [12-14, 19]. In the studies that supported EN, the mean tumor diameter was about $5 \mathrm{~cm}$, and most of them were smaller than $10 \mathrm{~cm}$ [12-14]. A few studies included patients with very large hemangiomas and had contradictory results [19]. Therefore, the choice of the open surgical method for hemangioma cannot be decided based on these studies. Given the controversial results in the past, there are currently no guidelines for the choice between EN and LR. This study was conducted to evaluate the outcomes of the two open procedures and explore the factors affecting the choice of the two methods.

\section{Methods}

\section{Patient selection}

The clinical data of 337 patients with $\mathrm{HH}$ confirmed by postoperative pathology in Department of General Surgery at Shenging Hospital of China Medical University from April 2014 to August 2020 was retrospectively collected. This study was approved by the Ethics Review Committee of Shengjing Hospital, and all patients or their authorizers gave written informed consent before the operation. The inclusion criteria were as follows: (i) patients only received open LR or EN; (ii) patients with Child-Pugh A; (iii) postoperative pathology confirmed to be cavernous hemangioma. The exclusion criteria: (i) patients underwent both EN and LR; (ii) laparoscopic or robotic surgery; (iii) accompanied by serious systemic diseases; (iv) patients received preoperative transarterial embolization or any other non-surgical treatment for $\mathrm{HH}$; (v) patients who underwent EN initially but were later converted to LR.

\section{Preoperative evaluation}

Preoperative evaluation included detailed clinical history, physical examination, laboratory tests, radiological investigations including ultrasound, contrast enhanced CT and/or magnetic resonance imaging, and indocyanine green 15-min retention test (ICGR15). For large tumors and those with a close relationship with intrahepatic blood vessels or the inferior vena cava, three-dimensional visualization was applied to evaluate the location of the tumor and its relationship with blood vessels. The peripherally located $\mathrm{HHs}$ were defined as $\mathrm{HHs}$ located in S2, 3, 4b, 5, and 6. The esions located in S1, 4a, 7, and 8 were defined as the centrally located HHs (Fig. 1). HHs near to or even compressing the trunk or main branches of the portal vein, hepatic vein or hepatic artery were regarded as tumors proximal to the massive vessels. Additional relevant tests such as gastroscopy were performed to exclude other digestive tract-related diseases in some patients.

\section{Surgical procedures and perioperative management}

The patients in both groups received general anesthesia with endotracheal intubation. A right subcostal incision or a right transabdominal rectus incision was used to enter the abdomen and liver mobilization was done according to the location of the tumor.

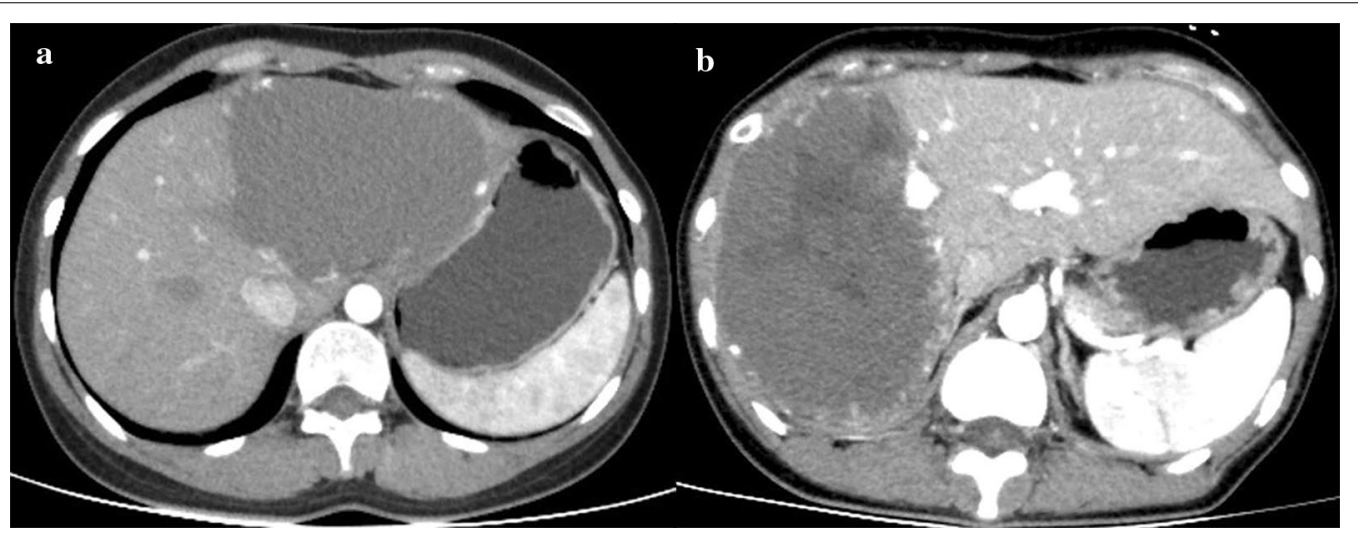

Fig. 1 Contrast-enhanced computed tomography of the abdomen: a A peripherally located hemangioma (S2 and S3) excised by EN. b A centrally located hemangioma (S7 and S8) excised by right hemihepatectomy 
1 EN-Dissection was performed along with the tumor capsule all around the tumor in the plane between the tumor and liver tissue without the loss of any normal hepatic parenchyma. The blood vessels and bile ducts entering or leaving the tumor were ligated and divided.

2 LR-For non-anatomical liver resection, the line of parenchymal transection was marked about $1-2 \mathrm{~cm}$ away from the tumor over the liver capsule using electrocautery. The liver parenchyma was divided with the classic clamp crushing technique. The larger intervening ducts and vessels of the liver were ligated and small diameter vessels were electrocoagulated until the liver tumor was completely removed.

In some cases, intermittent Pringle maneuver was used to reduce bleeding. Anatomical liver resection includes resection of Couinaud's segments, sectionectomy, and hemihepatectomy. An intraoperative ultrasound would be used to ensure complete resection of the tumor if necessary.

All patients received similar postoperative management. Ultrasound or CT scans were performed one week after the operation. The laboratory tests were examined every other day. The major perioperative complications were recorded and defined using the definition suggested by the International Study Group of Liver Surgery (ISGLS) [20, 21].

\section{Statistical analysis}

The continuous variables with normal distribution are expressed as mean \pm standard deviation $(S D)$, and the non-normal continuous variables are expressed as median (interquartile range). The independent sample $t$-test was used to compare the continuous normal distribution variables. The Mann-Whitney $U$ test was used to compare the continuous non-normal distribution variables. The Pearson Chi-square test or Fisher exact probability method was used to comparing the categorical data. Baseline variables with a $P<0.05$ on univariate analysis were included in the multivariate analysis. Logistic regression was performed to determine the independent factors associated with the choice of the surgical methods. Subsequently, the receiver operating characteristic (ROC) curve was graphed with the probabilities, and the area under the curve (AUC) was used to evaluate the efficacy of the multivariate combined model (Fig. 2). The calibration of the model was evaluated by the Hosmer-Lemeshow good of fit test. Statistical analysis was performed using SPSS 26.0 for Windows (SPSS Inc., Chicago, IL, USA). A $P$-value $<0.05$ was considered to be statistically significant.

\section{Propensity score matching (PSM)}

In this study, PSM was used to adjust the baseline difference between the EN and LR groups. PSM analysis was conducted with SPSS 26.0 for Windows (SPSS 26, Inc., Chicago, IL, USA). The propensity score of each patient was analyzed by multivariate logistic regression. The width of 0.2 caliper was selected and the matching ratio was $1: 1$. The nearest neighbor method was used to match the groups (Fig. 3).

\section{Results}

\section{Basic characteristics of patients}

A total of 337 patients underwent surgery for $\mathrm{HH}$ during the study period. According to the exclusion criteria, 16 patients who underwent both LR and EN, 39 patients who underwent laparoscopic or robotic surgery, 5 patients with severe systemic diseases, 3 patients who received preoperative non-surgical treatment for $\mathrm{HH}$ and one patient who was converted from EN to LR were excluded. The remaining 273 patients were divided into the EN $(n=163)$ and LR $(n=110)$ groups. The indications for surgery in this study were: (i) tumor diameter $\geq 5 \mathrm{~cm}$ with obvious symptoms such as upper abdominal discomfort or pressure symptoms $(\mathrm{n}=230)$; (ii) a rapid increase in a short term [annual growth $\geq 2 \mathrm{~cm}$ in diameter $(\mathrm{n}=25)$ ]; (iii) liver cancer could not be excluded $(\mathrm{n}=18)$.

In the EN group, there were 124 women $(76.1 \%)$ and 39 men $(22.9 \%)$ with a mean age of $47.3 \pm 9.0$ years and a mean body mass index (BMI) of $23.3 \pm 3.5 \mathrm{~kg} / \mathrm{m}^{2}$. Twenty-four patients (14.7\%) had fatty liver and three (1.8\%) had liver cirrhosis. There were 64 patients with lesions near the major vessels (39.3\%) and 142 patients had peripherally located lesions (87.1\%). The mean diameter of the tumor was $9.4 \pm 2.8 \mathrm{~cm}$. A solitary tumor was observed in 97 patients (59.5\%) and multiple tumors were observed in 66 patients (40.5\%).

In the LR group, there were 79 women $(71.8 \%)$ and 31 men (28.2\%) with a mean age of $49.0 \pm 10.2$ years and a mean BMI of $23.4 \pm 3.0 \mathrm{~kg} / \mathrm{m}^{2}$. Twenty-two patients (20.0\%) had fatty liver and four (3.6\%) had liver cirrhosis. There were 30 patients with lesions close to the major blood vessels $(27.3 \%)$ and 52 patients with peripherally located lesions (42.7\%). The mean diameter of the tumor was $11.5 \pm 4.2 \mathrm{~cm}$. A single tumor was seen in 83 patients (75.5\%) and multiple tumors were observed in 27 patients (24.5\%).

After PSM of age, sex, BMI, presence of liver cirrhosis or fatty liver, tumor location (proximal to massive vessels or not), tumor distribution (centrally located or peripherally located), tumor size and tumor number, 66 patients from each group were included (Table 1, Fig. 4). 

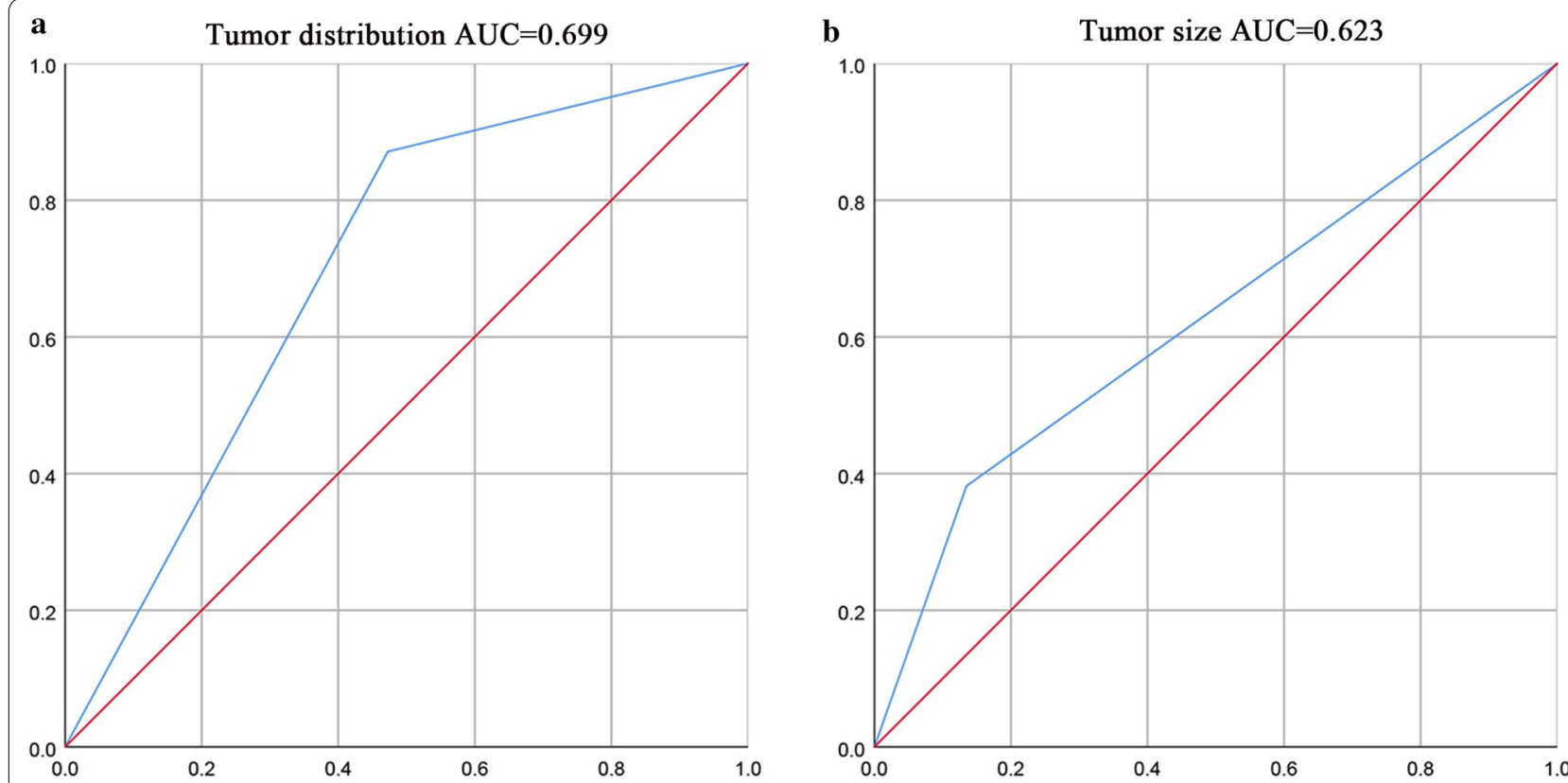

c

Tumor number $\mathrm{AUC}=\mathbf{0 . 5 8 0}$

d

The combined variables $\mathrm{AUC}=0.781$
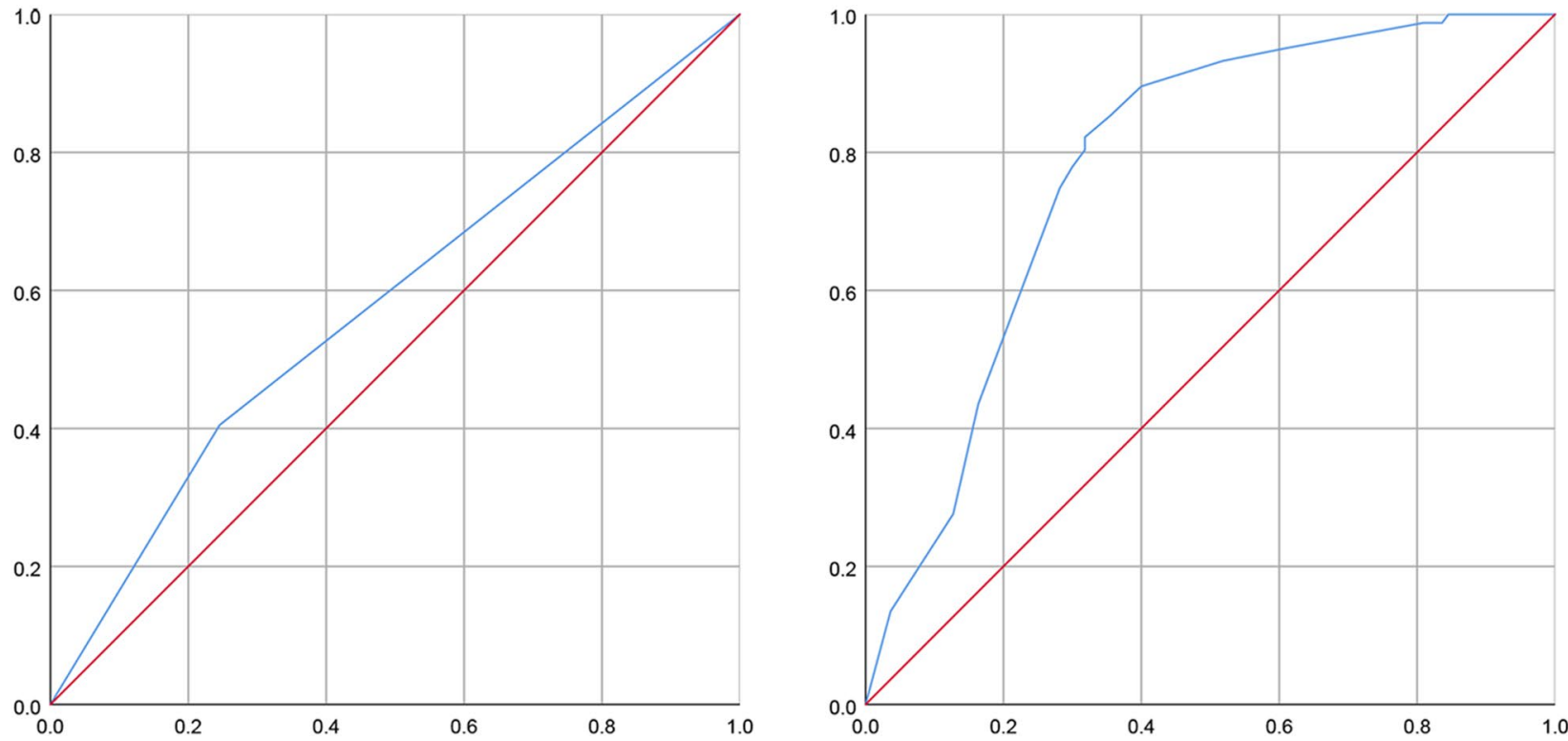

Fig. 2 Predictive efficacy of the three variables and the combined variable. The AUCs for the choice of the surgical methods were $0.699,0.623,0.580$ for the variables: tumor distribution, tumor diameter, and tumor number, respectively $(\mathbf{a}-\mathbf{c})$. Furthermore, the AUC of the combined variables was $0.781(\mathbf{d})$

\section{Independent factors affecting the choice of the surgical methods}

As shown in Table 1, the four baseline variables, namely, tumor location, tumor distribution, tumor number, and tumor size were unbalanced before matching. To determine the factors affecting the choice of surgical procedure for $\mathrm{HH}$, we included these variables in the univariate analysis. Previously, tumor size was transformed into binary variables based on the cut-off value that was revealed by the ROC curve (Youden index: 0.2468 ; associated criterion: $\leq 12.1 \mathrm{~cm}$; sensitivity: 86.50\%; specificity: $36.18 \%$ ). The surgical method was taken as the dependent variable $(\mathrm{LR}=0, \mathrm{EN}=1)$ which meant that EN was taken as the experimental group 
a
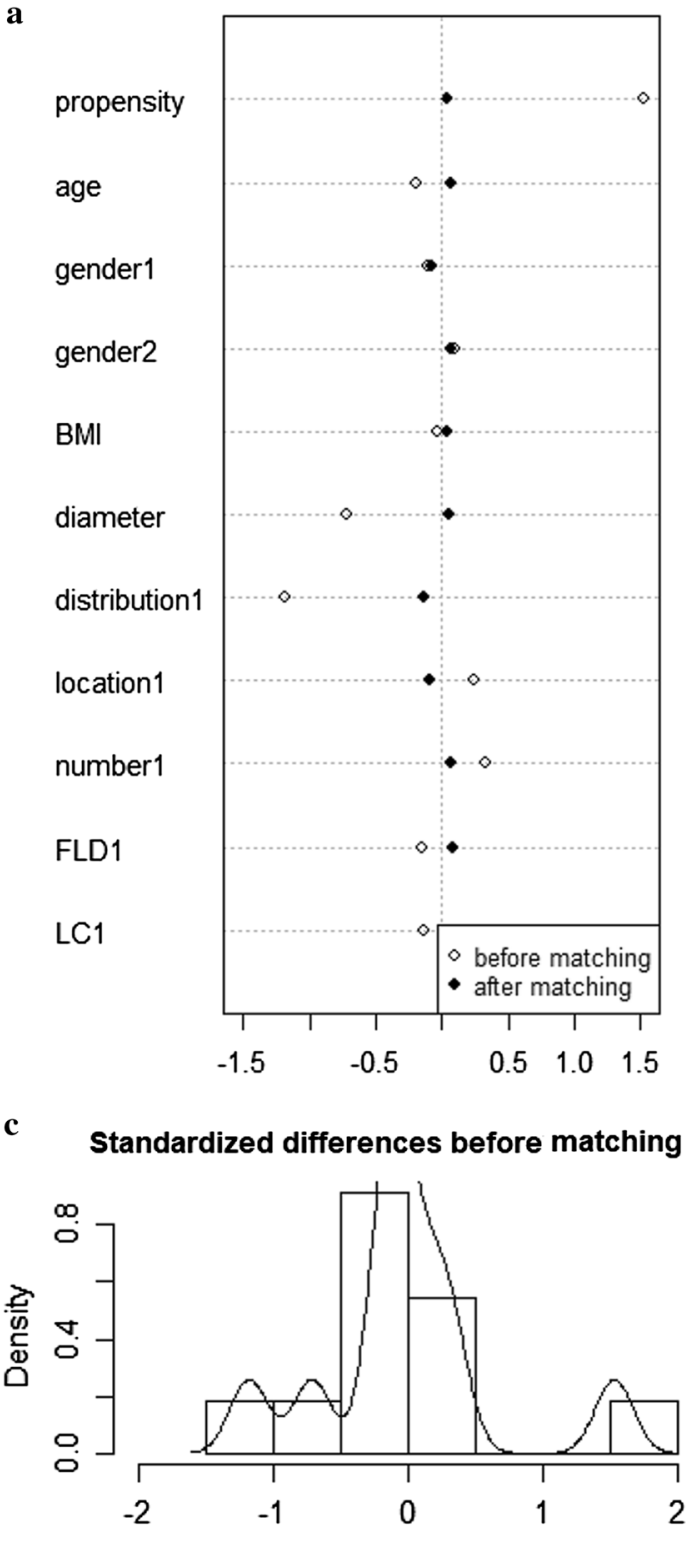

b

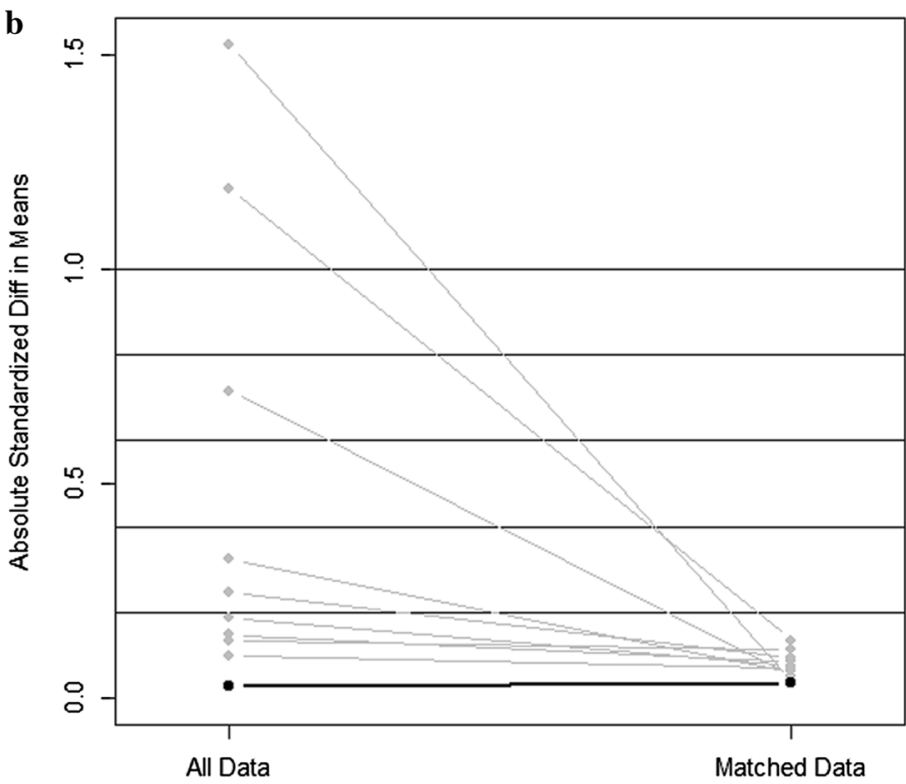

Std. difference

Fig. 3 Plot of the propensity score-matched study. a Dot plot of standardized mean difference, $\mathbf{b}$ line plot of individual differences, $\mathbf{c}$ histogram of standardized mean differences (before and after)

and LR as the control group. Other related factors (tumor location: not proximal to the major vessels $=0$, proximal to the major vessels $=1$; tumor distribution: peripherally located $=0$, centrally located $=1$; tumor size: $\leq 12.1 \mathrm{~cm}=0,>12.1 \mathrm{~cm}=1$; tumor number: single $=0$, multiple $=1$ ) were taken as independent variables or exposure factors. OR was equal to the ratio of exposure to non-exposure in EN group divided by the ratio of exposure to non-exposure in LR group. On univariate analysis with $\alpha=0.05$ as the test level, lesions proximal to the major vessels [OR: 1.724, 95\% confidence interval (CI) 1.020-2.912] (lesions proximal to the major vessels were 1.724 times more likely to undergo EN than those far away from major vessels), centrally located lesions (OR: 0.133, 95\% CI 0.0730.240 ) (centrally located lesions were 0.133 times less likely to undergo EN than peripherally located lesions), tumors size $>12.1 \mathrm{~cm}$ (OR: 0.253 , 95\% CI 0.140-0.456) (tumors > 12.1 were 0.253 times less likely to undergo EN than tumors $\leq 12.1 \mathrm{~cm}$ ) and multiple tumors (OR: 
Table 1 Demographics, comorbidities, and pre-operative data for patients undergoing surgery for hepatic hemangioma

\begin{tabular}{|c|c|c|c|c|c|c|}
\hline \multirow[t]{2}{*}{ Variable } & \multicolumn{3}{|c|}{ Before propensity } & \multicolumn{3}{|c|}{ After propensity } \\
\hline & EN & LR & $P$ value & EN & LR & $P$ value \\
\hline Number of patients (\%) & $163(100)$ & $110(100)$ & & $66(100)$ & $66(100)$ & \\
\hline Age (years) & $47.3 \pm 9.0$ & $49.0 \pm 10.2$ & 0.154 & $47.6 \pm 9.4$ & $47.0 \pm 9.0$ & 0.693 \\
\hline \multicolumn{7}{|l|}{ Gender } \\
\hline Female & $124(76.1)$ & $79(71.8)$ & 0.481 & $50(75.8)$ & $48(72.7)$ & 0.691 \\
\hline Male & $39(22.9)$ & $31(28.2)$ & & $16(24.2)$ & $18(27.3)$ & \\
\hline BMI $\left(\mathrm{kg} / \mathrm{m}^{2}\right)$ & $23.3 \pm 3.5$ & $23.4 \pm 3.0$ & 0.817 & $23.4 \pm 3.6$ & $23.2 \pm 2.9$ & 0.827 \\
\hline \multicolumn{7}{|l|}{ Comorbidities } \\
\hline Fatty liver & $24(14.7)$ & $22(20.0)$ & 0.253 & $13(19.7)$ & $11(16.7)$ & 0.652 \\
\hline Liver cirrhosis & $3(1.8)$ & $4(3.6)$ & 0.445 & $3(4.5)$ & $2(3.0)$ & 1.000 \\
\hline \multicolumn{7}{|l|}{ Preoperative data } \\
\hline Tumor proximal to massive vessels & & & $0.041^{*}$ & & & 0.561 \\
\hline Yes & $64(39.3)$ & $30(27.3)$ & & $17(25.8)$ & $20(30.3)$ & \\
\hline No & $99(60.7)$ & $80(72.7)$ & & $49(74.2)$ & $46(69.7)$ & \\
\hline Tumor distribution & & & $<0.001^{*}$ & & & 0.573 \\
\hline Centrally located & $21(12.9)$ & $58(42.7)$ & & $19(28.8)$ & $22(33.3)$ & \\
\hline Peripherally located & $142(87.1)$ & $52(57.3)$ & & $47(71.2)$ & $44(66.7)$ & \\
\hline Tumor size (cm) & $9.4 \pm 2.8$ & $11.5 \pm 4.2$ & $<0.001^{*}$ & $10.2 \pm 2.9$ & $10.1 \pm 3.5$ & 0.802 \\
\hline Tumor number & & & $0.006^{*}$ & & & \\
\hline Single & $97(59.5)$ & $83(75.5)$ & & $42(63.6)$ & $44(66.7)$ & 0.715 \\
\hline Multiple & $66(40.5)$ & $27(24.5)$ & & $24(36.4)$ & $22(33.3)$ & \\
\hline
\end{tabular}

Before propensity score matching, there were significant differences between the two groups in the location related to massive vessels, tumor distribution, radiographic tumor size, and tumor number between group $E(n=163)$ and group $L R(n=110)$. After matching, the four baseline variables were balanced $(P>0.05)$ $L R$ Liver resection, EN Enucleation, $B M I$ Body mass index

${ }^{*} P<0.05$ was considered significant difference

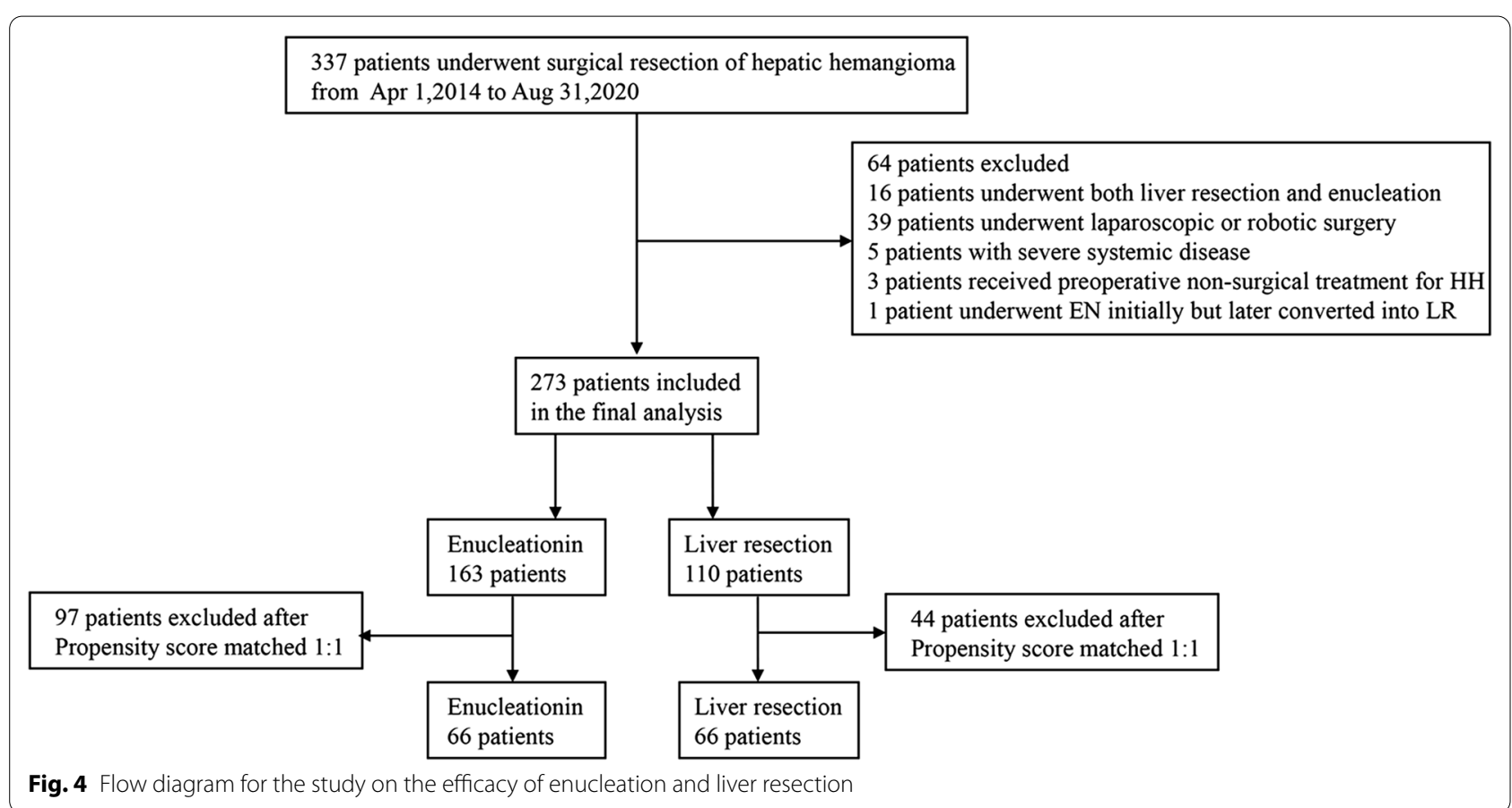


2.092, 95\% CI 1.225-3.572) (multiple tumors were 2.092 times more likely to undergo EN than single tumors) were found to affect the choice of surgical method in univariate analysis and were included in multivariate logistic regression. The final results showed that centrally located lesions (OR: 0.131, 95\% CI 0.070-0.244), tumors size > $12.1 \mathrm{~cm}$ (OR: $0.226,95 \%$ CI $0.116-0.439$ ) and multiple tumors (OR: 1.860, 95\% CI 1.003-3.449) were independent factors affecting the choice of EN (Table 2). With these identified independent factors, the ROC was graphed independently for each variable and the combined variable (Fig. 2). The calibration of the model was evaluated by the Hosmer-Lemeshow good of fit test $\left(\chi^{2}=12.174, p=0.095\right)$.

Table 2 Univariate and multivariate Logistic regression analysis

\begin{tabular}{|c|c|c|c|c|}
\hline \multirow[t]{2}{*}{ Factors included } & \multicolumn{2}{|c|}{$\begin{array}{l}\text { Univariate } \\
\text { Logistic }\end{array}$} & \multicolumn{2}{|c|}{$\begin{array}{l}\text { Multivariate } \\
\text { logistic }\end{array}$} \\
\hline & $O^{\wedge} R$ & $95 \% \mathrm{Cl}$ & $\mathrm{O}^{\wedge} \mathrm{R}$ & $95 \% \mathrm{Cl}$ \\
\hline Proximal to the major vessels & 1.724 & $1.020-2.912$ & 1.599 & $0.869-2.940$ \\
\hline Centrally located lesions & 0.133 & $0.073-0.240$ & 0.131 & $0.070-0.244$ \\
\hline Tumors size $>12.1 \mathrm{~cm}$ & 0.253 & $0.140-0.456$ & 0.226 & $0.116-0.439$ \\
\hline Multiple tumors & 2.092 & $1.225-3.572$ & 1.860 & $1.003-3.449$ \\
\hline \multicolumn{5}{|c|}{$\begin{array}{l}\text { EN was taken as the experimental group and LR as the control group. Proximal } \\
\text { to the major vessels, centrally located, tumor size }>12.1 \mathrm{~cm} \text { and multiple tumors } \\
\text { were taken as independent variables or exposure factors. OR was equal to the } \\
\text { ratio of exposure to non-exposure in EN group divided by the ratio of exposure } \\
\text { to non-exposure in LR group }\end{array}$} \\
\hline
\end{tabular}

Comparison of the perioperative outcomes of two groups after PSM

There was no significant difference in median operative time (156 min vs. $195 \mathrm{~min}$ ), median estimated blood loss $(200 \mathrm{ml}$ vs. $220 \mathrm{ml})$, or the blood transfusion rate $(33.3 \%$ vs. 33.3\%) between the EN and LR groups. There was no significant difference in the mean postoperative feeding (3.1 d vs. $3.3 \mathrm{~d}$ ), mean abdominal drain duration (5.9 d vs. $6.0 \mathrm{~d}$ ), or mean postoperative hospital stay (9.5 d vs. 9.0 d) between the two groups. Postoperative complications including ascites (6.1\% vs. $4.5 \%)$, abdominal infection (6.1\% vs. $4.5 \%)$, biliary fistula ( $1.5 \%$ vs. $3.0 \%)$ and postoperative bleeding (1.5 vs. $4.5 \%$ ) were comparable between the two groups (Table 3 ). There were no perioperative deaths.

\section{Discussion}

Based on the univariate and multivariate analysis, we found that tumor size $>12.1 \mathrm{~cm}$, centrally located lesions, and multiple tumors were independent factors affecting the choice of EN. After PSM, there were no significant differences in the efficacy of EN or LR.

In the pre-operative data, we found that tumor size in EN group was significantly smaller than that in LR group which suggests that LR was preferred for giant hemangiomas, especially for $\mathrm{HH}$ sized $>12.1 \mathrm{~cm}$. Giant $\mathrm{HH}$ occupies more than one liver segment, so the cut surface is wide and the risk of bleeding is high after enucleation. Moreover, once the capsule is injured during EN, the bleeding is difficult to control. On the contrary, during anatomical LR, the blood vessels supplying the liver segment or lobe are ligated first, which ensures the surgical

Table 3 Peri-operative data for patients undergoing surgical resection of hepatic hemangioma in this cohort

\begin{tabular}{|c|c|c|c|c|c|c|}
\hline \multirow[t]{2}{*}{ Variable } & \multicolumn{3}{|c|}{ Before propensity } & \multicolumn{3}{|c|}{ After propensity } \\
\hline & EN & LR & $P$ value & $\overline{E N}$ & LR & $P$ value \\
\hline Number of patients (\%) & $163(100)$ & $110(100)$ & & $66(100)$ & $66(100)$ & \\
\hline Operation time (minutes) & $150(130-200)$ & 195 (149-246) & $<0.001^{*}$ & $156(130-210)$ & 195 (139-238) & 0.156 \\
\hline Estimated blood loss (ml) & $200(100-300)$ & $300(100-450)$ & $0.048^{*}$ & $200(150-425)$ & $220(100-360)$ & 0.423 \\
\hline Blood transfusion rate & $37(22.7)$ & $38(34.5)$ & $0.031^{*}$ & $22(33.3)$ & $22(33.3)$ & 1.000 \\
\hline \multicolumn{7}{|l|}{ Postoperative data } \\
\hline Postoperative feeding (days) & $3.2 \pm 1.2$ & $3.5 \pm 1.3$ & $0.027^{*}$ & $3.1 \pm 1.0$ & $3.3 \pm 1.0$ & 0.460 \\
\hline Abdominal drains (days) & $5.8 \pm 1.9$ & $6.2 \pm 2.6$ & $0.036^{*}$ & $5.9 \pm 1.9$ & $6.0 \pm 1.9$ & 0.643 \\
\hline Postoperative hospital stay (days) & $9.1 \pm 2.5$ & $9.2 \pm 3.3$ & 0.700 & $9.5 \pm 2.6$ & $9.0 \pm 1.9$ & 0.206 \\
\hline \multicolumn{7}{|l|}{ Complication } \\
\hline Ascites & $7(4.3)$ & $5(4.5)$ & 1.000 & $4(6.1)$ & $3(4.5)$ & 1.000 \\
\hline Abdominal infection & $10(6.1)$ & $10(9.1)$ & 0.358 & $4(6.1)$ & $3(4.5)$ & 1.000 \\
\hline Bile fistula & $1(0.6)$ & $5(4.5)$ & $0.041^{*}$ & $1(1.5)$ & $2(3.0)$ & 1.000 \\
\hline Bleeding & $2(1.1)$ & $4(2.8)$ & 0.224 & $1(1.5)$ & $3(4.5)$ & 0.619 \\
\hline
\end{tabular}

$L R$ liver resection, $E N$ enucleation

${ }^{*} P<0.05$ was considered significant difference 
safety. Liu et al. proposed in a retrospective study that large hemangiomas, especially those extremely giant liver hemangioma $>20 \mathrm{~cm}$ were more suitable for LR because of less use of Pringle maneuver [19]. At the same time, due to the complete preservation of the vessels of the remaining liver, the function of the remnant liver is preserved. Borgonovo et al. [17] deemed that to achieve lower morbidity and blood loss, in the case of large HHs, typical liver resection was the best choice and EN was an option when a lesion was small. However, it is undeniable that anatomical LR requires higher surgical skills, so only experienced surgeons who can deal with complex blood vessels and anatomical variations should undertake LR.

EN may be preferred for lesions located peripherally where the intraoperative exposure was relatively sufficient to allow EN. The centrally distributed HHs embedded in the liver parenchyma are difficult to remove with EN. For example, HH occupying S7 and S8 was very deep and close to the top of the diaphragm, so the patient received anatomical right hemihepatectomy and was discharged successfully without any complications (Fig. 1). Orhan et al. considered that if such tumor location as mentioned above precludes safe $\mathrm{EN}$, anatomic resection is preferred [22].

In this study, patients with multiple $\mathrm{HH}$ were more inclined to receive EN. This was because the multiple lesions were widely distributed and LR would have resulted in more loss of liver parenchyma, and a higher incidence of postoperative liver failure and other complications [22]. But if the multiple tumors were located in the same or adjoining liver segments, it was easier to perform LR along anatomical planes rather than performing $\mathrm{EN}$ for each lesion. In some cases, if multiple HHs are widely distributed (distributed in different hepatic lobes and segments), then EN may be combined with LR, or a multi-stage operation can be performed if the future liver remnant (FLR) is insufficient.

Since the first use of EN in 1988, many studies found EN to have better outcomes for $\mathrm{HH}[12-15,23]$. There are fewer blood vessels along with the capsule so the vascular and bile duct injury is avoided to the greatest extent $[24,25]$. Also, the normal liver tissue is preserved as much as possible, there by reducing the risk of postoperative liver dysfunction [19]. In this study, EN was indeed superior to LR, in terms of peri-operative data such as operative time, estimated blood loss, postoperative feeding, abdominal drains, and postoperative hospital stay before PSM. However, after PSM the outcomes of EN and LR groups were similar. Zhang et al. [26] in their study of 86 cases of $\mathrm{HH}$ with a diameter $>10 \mathrm{~cm}$ found that there was no significant difference in operative time, hepatic inflow occlusion, blood loss or complications between the two groups. It's worth noting that these previous studies had some limitations: the sample size was small and there was no separate analysis of the impact of the size, location and number of $\mathrm{HHs}$ on the choice of surgical methods. In this study, we believe that it was unreasonable to statistically analyze the non-comparable data of two groups. So we applied PSM, a method that could eliminate the confounding bias and make the study more scientific. Each procedure has its own advantages and disadvantages. As long as we reasonably master the technique of EN and LR instead of blindly pursuing a certain surgical method, we could use both these procedures depending the tumor size, location and number in order to the best outcomes.

There are some limitations to this study. First, this is a retrospective single-center study instead of an intention-to-treat (ITT) analysis and the choice of surgical procedure may be influenced by the experience of surgeons. Second, the number of patients in each group after PSM was relatively small. Hence, future prospective studies with larger sample sizes and long-term follow-up are needed to further validate our results.

\section{Conclusions}

Patients with symptomatic giant HHs having peripherally located lesions, tumors size less than or equal to $12.1 \mathrm{~cm}$ and multiple tumors were more inclined to receive EN by open surgery. After PSM analysis, there was no enough evidence to prove that there were significant differences in the efficacy of EN or LR. To give full play to the superiority of the two surgical methods, above-mentioned factors must be taken into account.

\section{Abbreviations}

HH: Hepatic hemangioma; LR: Liver resection; EN: Enucleation; PSM: Propensity score matching; CT: Computed tomography; MRI: Magnetic resonance imaging; FLR: Future liver remnant; ICGR15: Indocyanine green 15-min retention test; BMI: Body mass index; AUC: Area under the curve; ROC: Receiver operating characteristic; OR: Odds ratio; FLD: Fatty liver disease; LC: Liver cirrhosis.

\section{Acknowledgements}

Mingguang Ju wants to thank, in particular, the inimitable care and support from Heyue Zhang over the past months.

\section{Authors' contributions}

JMG, XF, ZWY - Study concept and design, acquisition of data, analysis, and interpretation of data, drafting of the manuscript, revision of the manuscript. $\mathrm{DCL}$ - Interpretation of data, contributed to the discussion and final approval of the manuscript, ensuring that the entire author group is fully aware of and in compliance with best practices in the discipline of publication. All authors read and approved the final manuscript.

\section{Funding}

This work was supported by the key R\&D projects of Liaoning Province [Grant numbers 2017225032]; Shenyang Science and Technology Project [Grant number 17-230-9-16]. Corresponding author DCL is the guarantor. The funding body financed the costs of the study and contributed to the design of the study, interpretation of data, and revising the manuscript. 


\section{Availability of data and materials}

The datasets used and analyzed during the current study are available from the corresponding author on reasonable request.

\section{Ethics approval and consent to participate}

This study was approved by the Ethics Review Committee of Shengjing Hospital (2019PS673K) and was performed in accordance with the Declaration of Helsinki. All patients or their authorizers gave written informed consent before the operation.

\section{Consent for publication}

Written informed consent was obtained from the patients and/or their legal guardians for publication, and any accompanying images, sex, age of these patients

\section{Competing interests}

No benefits in any form have been received or will be received from a commercial party related directly or indirectly to the subject of this article.

\section{Received: 30 July 2020 Accepted: 28 October 2020}

Published online: 07 November 2020

\section{References}

1. Adam YG, Huvos AG, Fortner JG. Giant hemangiomas of the liver. Ann Surg. 1970;172(2):239-45. https://doi.org/10.1097/00000658-19700800000010.

2. Leon M, Chavez L, Surani S. Hepatic hemangioma: what internists need to know. World J Gastroenterol. 2020;26(1):11-20. https://doi.org/10.3748/ wjg.v26.i1.11.

3. Oak CY, Jun CH, Cho EA, et al. Hepatic hemangioma with Kasabach-Merritt syndrome in an adult patient. Korean J Gastroenterol. 2016;67(4):2203. https://doi.org/10.4166/kjg.2016.67.4.220.

4. Albitar HA, lyer V. Giant liver hemangioma with Kasabach-Merritt syndrome. Am J Med. 2019. https://doi.org/10.1016/j.amjmed.2019.06.024.

5. Moctezuma-Velazquez C, Lopez-Arce G, Martinez-Rodriguez LA, et al. Giant hepatic hemangioma versus conventional hepatic hemangioma: clinical findings, risk factors, and management. Rev Gastroenterol Mex. 2014;79(4):229-37. https://doi.org/10.1016/j.rgmx.2014.08.007.

6. Weimann A, Ringe B, Klempnauer J, et al. Benign liver tumors: differential diagnosis and indications for surgery. World J Surg. 1997;21(9):983-90. https://doi.org/10.1007/s002689900337 (discussion 990-981).

7. Liu X, Yang Z, Tan H, et al. Characteristics and operative treatment of extremely giant liver hemangioma $>20 \mathrm{~cm}$. Surgery. 2017;161(6):151424. https://doi.org/10.1016/j.surg.2016.12.015.

8. Di Carlo I, Koshy R, Al Mudares S, et al. Giant cavernous liver hemangiomas: is it the time to change the size categories? Hepatobiliary Pancreat Dis Int. 2016;15(1):21-9.

9. Qu C, Liu H, Li XQ, et al. Percutaneous ultrasound-guided "three-step" radiofrequency ablation for giant hepatic hemangioma $(5-15 \mathrm{~cm})$ : a safe and effective new technique. Int J Hyperthermia. 2020;37(1):212-9. https ://doi.org/10.1080/02656736.2020.1732484.

10. Dindo D, Demartines N, Clavien PA. Classification of surgical complications: a new proposal with evaluation in a cohort of 6336 patients and results of a survey. Ann Surg. 2004;240(2):205-13. https://doi. org/10.1097/01.sla.0000133083.54934.ae.
11. Alper A, Ariogul O, Emre A, et al. Treatment of liver hemangiomas by enucleation. Arch Surg. 1988;123(5):660-1. https://doi.org/10.1001/archs urg.1988.01400290146027.

12. Singh RK, Kapoor S, Sahni P, et al. Giant haemangioma of the liver: is enucleation better than resection? Ann R Coll Surg Engl. 2007;89(5):490-3. https://doi.org/10.1308/003588407×202038.

13. Liu $Y$, Wei $X$, Wang $K$, et al. Enucleation versus anatomic resection for giant hepatic hemangioma: a meta-analysis. Gastrointest Tumors. 2017;3(34):153-62. https://doi.org/10.1159/000455846.

14. Cheng WL, Qi YQ, Wang B, et al. Enucleation versus hepatectomy for giant hepatic haemangiomas: a meta-analysis. Ann R Coll Surg Engl. 2017;99(3):237-41. https://doi.org/10.1308/rcsann.2016.0349.

15. Wu CX, Bao WY, Zhu F. Enucleation is better for a giant hemangioma proximal to the hepatic portal vascular structures. Indian J Surg. 2016;78(4):304-8. https://doi.org/10.1007/s12262-015-1382-9.

16. Hamaloglu E, Altun $\mathrm{H}$, Ozdemir A, et al. Giant liver hemangioma: therapy by enucleation or liver resection. World J Surg. 2005;29(7):890-3. https:// doi.org/10.1007/s00268-005-7661-z.

17. Borgonovo G, Razzetta F, Arezzo A, et al. Giant hemangiomas of the liver: surgical treatment by liver resection. Hepatogastroenterology. 1997;44(13):231-4.

18. Abdel WM, Nakeeb A, Ali MA, et al. Surgical management of giant hepatic hemangioma: single center's experience with 144 patients. J Gastrointest Surg. 2018;22(5):849-58. https://doi.org/10.1007/s1 1605-018-3696-y.

19. Liu XL, Yang ZY, Haidong T, et al. Characteristics and operative treatment of extremely giant liver hemangioma $>20 \mathrm{~cm}$. Surgery. 2017;161(6):151424. https://doi.org/10.1016/j.surg.2016.12.015

20. Rahbari NN, Garden OJ, Padbury R, et al. Posthepatectomy liver failure: a definition and grading by the International Study Group of Liver Surgery (ISGLS). Surgery. 2011;149(5):713-24. https://doi.org/10.1016/j. surg.2010.10.001.

21. Koch M, Garden OJ, Padbury R, et al. Bile leakage after hepatobiliary and pancreatic surgery: a definition and grading of severity by the International Study Group of Liver Surgery. Surgery. 2011;149(5):680-8. https:// doi.org/10.1016/j.surg.2010.12.002.

22. Chuang YH, Ou HY, Lazo MZ, et al. Predicting post-hepatectomy liver failure by combined volumetric, functional MR image and laboratory analysis. Liver Int. 2018;38(5):868-74. https://doi.org/10.1111/liv.13608.

23. Kuo PC, Lewis WD, Jenkins RL. Treatment of giant hemangiomas of the liver by enucleation. J Am Coll Surg. 1994;178(1):49-53.

24. De WE, Ettorre GM, Levi Sandri GB, et al. Major hepatectomy for a symptomatic giant liver cavernous hemangioma. Hepatobiliary Surg Nutr. 2015;4(3):218-9. https://doi.org/10.3978/j.issn.2304-3881.2015.01.08.

25. Machado MAC, Surjan R, Basseres T, et al. Laparoscopic parenchymalsparing liver resections using the intrahepatic glissonian approach. Ann Surg Oncol. 2017;24(8):2353-4. https://doi.org/10.1245/s1043 4-017-5886-3.

26. Zhang W, Huang ZY, Ke CS, et al. Surgical treatment of giant liver hemangioma larger than $10 \mathrm{~cm}$ : a single center's experience with 86 patients. Medicine. 2015;94(34):e1420. https://doi.org/10.1097/md.0000000000 001420.

\section{Publisher's Note}

Springer Nature remains neutral with regard to jurisdictional claims in published maps and institutional affiliations. 